\title{
Comparative Analysis of SSRLS and SSRLS with Adaptive Memory for Wireless Channel Equalization
}

\author{
Muhammad Zeeshan and Ihsan Ullah
}

\begin{abstract}
Wireless radio channels cause severe distortion in the signals. These distortions can be reduced by using different channel equalization techniques. In this paper, we compare the equalization performance of state-space recursive least squares (SSRLS) and state-space recursive least squares with adaptive memory (SSRLSWAM) to offset the effect of a linear dispersive channel. These adaptive filters are well-suited for the estimation of deterministic signals corrupted by the observation noise. We consider the equalization of both the linear time invariant and linear time varying systems to compare the performance of these filters. The performance is affected by the observation noise variance, choice of the forgetting factor and the model mismatch. We present simulation results to compare these cases by tracking a deterministic signal. It has been shown that SSRLSWAM outperforms SSRLS in both the time invariant and time varying channels.
\end{abstract}

Index Terms-SSRLS, SSRLSWAM, adaptive channel equalization, tracking, radio channels

\section{INTRODUCTION}

Adaptive filters have the ability to track time variations of input statistics by operating in environments with model uncertainties and unknown noise variances. This ability makes adaptive filters a very promising tool for signal processing, radar, communication, biomedical engineering applications. All these applications have one basic function is common; an estimation error is computed by using an input signal and a desired signal. This estimation error in turn is used to control values of the tunable coefficients of the adaptive filter [1].

Inverse modeling is an important class of adaptive filtering applications. In this class, the adaptive filter provides an inverse model that represents the best fit to an unknown noisy model. In this project, our work is also based on this class of adaptive filters.

Adaptive channel equalization is the process of estimating the original input to the channel by continuously adapting its adjustable coefficients. An adaptive channel equalizer can be implemented to perform the tap weight adjustments periodically or continually [2]. In this way, the effect of a linear dispersive channel on a signal propagating through it can be offset.

Manuscript received November 14, 2012; revised January 22, 2013.

Muhammad Zeeshan is with the Department of Electrical Engineering, College of Electrical and Mechanical Engineering, National University of Sciences and Technology, Pakistan (e-mail: ranazeeshan@ceme.nust.edu.pk).

Ihsan Ullah is with the Department of Electrical Engineering, Hanyang University, South Korea (e-mail: engr.ihsan@hotmail.com).
State-space recursive least-squares (SSRLS) is able to estimate many deterministic signals corrupted by observation noise [3]. It is not just a state-space representation of the standard RLS filter rather it has better tracking performance as compared to the standard RLS filter. One of the challenges faced by SSRLS is to track a time-varying signal with unknown underlying system model. SSRLS filters face some limitations in this time varying case. To overcome this difficulty, we use SSRLS with adaptive memory (SSRLSWAM) which is an extension of the SSRLS [4]. This filter works on the fact that the forgetting factor becomes a function of time, and thus it adapts itself accordingly.

In this paper, we first describe the channel impulse response. In Section III and IV, we describe the algorithms of SSRLS and SSRLSWAM filters to be used for the equalization of this channel. The model used for the simulation of these algorithms has been presented in Section V. Simulation results are given in Section VI followed by conclusion and references in Section VII.

\section{WIRELESS CHANNEL IMPULSE RESPONSE}

The wireless channels are mostly modeled by a Tapped Delay Line (TDL) model or Finite Impulse Response (FIR) filter. The result of transmission through the channel is the severe distortion in the amplitude and phase of the signal. In this work, we have to performed adaptive channel equalization using SSRLS and SSRLSWAM algorithms on a known channel modeled by a 12-tap linear transversal filter. The input to the filter is a deterministic signal. For the time invariant case, the impulse response of this filter is given by;

$$
h[n]= \begin{cases}\frac{1}{2}\left[1+\cos \left(\frac{2 \pi}{3}(n-2)\right)\right], & n=0,1, \ldots ., 11 \\ 0 & \text { otherwise }\end{cases}
$$

The output of the channel is corrupted by Gaussian observation noise of zero mean and variance $\sigma_{v}{ }^{2}$. As in the case of a practical communication system, the equalizer has no knowledge about the channel order, the coefficient values and observation noise variance. But we have assumed that perfect channel estimation is available. Following two cases have been discussed in this paper;

\section{A. Case 1}

In this case, the channel is time invariant and we have to perform non-blind equalization i.e. the equalizer has access to the input and noise corrupted output of the channel. 


\section{B. Case 2}

In this case, the channel is time varying and again we have to perform non-blind equalization. The variations of the time varying tap weights are very slow as compared to the variations of the input signal.

\section{SSRLS ALGORITHM}

In this section, we describe the summarized algorithm of SSRLS which has been used for the estimation of deterministic signal corrupted by observation noise and wireless channel. Since it is a recursive algorithm, it needs to be initialized. The method of regularization term has been used for initialization. Following initializations can be taken to simplify the process.

$$
\psi[0]=\delta \mathbf{I}(\text { for } \delta>0), \hat{x}[0]=\mathbf{0}
$$

The algorithm then proceeds as follows.

Predicted (a-priori) states

$$
\bar{x}_{k}=A \hat{x}_{k-1}
$$

Predicted output (Predicted received signal)

$$
\bar{y}_{k}=C \bar{x}_{k}
$$

Prediction error (Received signal $y_{k}$ is the input to the equalizer)

$$
\xi_{k}=y_{k}-\bar{y}_{k}
$$

Calculation of matrix $\Phi_{k}$

$$
\Phi_{k}=\lambda A^{-T} \Phi_{k-1} A^{-1}+C^{T} C
$$

Calculation of the inverse of matrix $\Phi_{k}$

$$
\begin{aligned}
\Phi_{k}^{-1}= & \lambda^{-1} A \Phi_{k-1}^{-1} A^{T}-\lambda^{-2} A \Phi_{k-1}^{-1} A^{T} C^{T} \\
& \times\left[I+\lambda^{-1} C A \Phi_{k-1}^{-1} A^{T} C^{T}\right]^{-1} \times C A \Phi_{k-1}^{-1} A^{T}
\end{aligned}
$$

Calculation of SSRLS gain

$$
K_{m}=\Phi_{k-1}^{-1} C^{T}
$$

States (a-posteriori) estimates

$$
\hat{x}_{k}=A \hat{x}_{k-1}+K_{k} \xi_{k}
$$

Output estimate ( $y_{k}$ is the equalized signal)

$$
\hat{y}_{k}=C \hat{x}_{k}
$$

Calculation of the estimation error for performance analysis

$$
e_{k}=y_{k}-\hat{y}_{k}
$$

\section{SSRLSWAM ALGORITHM}

In this section, we describe the summarized algorithm of SSRLS which has been used for the estimation of deterministic signal corrupted by observation noise and wireless channel. The major difference of this algorithm when compared to standard SSRLS is the tuning of the forgetting factor. Like SSRLS, it is also a recursive algorithm, so it needs to be initialized. The method of regularization term has been used for initialization. Following initializations can be taken to simplify the process.

$$
\psi[0]=\mathbf{0}, \hat{x}[0]=\mathbf{0}
$$

The algorithm then proceeds as follows.

Predicted (a-priori) states

$$
\bar{x}_{k}=A \hat{x}_{k-1}
$$

Predicted output (Predicted received signal)

$$
\bar{y}_{k}=C \bar{x}_{k}
$$

Prediction error (Received signal $y_{k}$ is the input to the equalizer)

$$
\xi_{k}=y_{k}-\bar{y}_{k}
$$

Calculation of matrix $\Phi_{k}$

$$
\lambda_{k}=\left[\lambda_{k-1}+\alpha \psi_{k-1} A^{T} C^{T} \xi_{k}\right]_{\lambda^{-}}^{\lambda^{+}}
$$

Calculation of SSRLS gain

$$
K_{k}=\lambda_{k-1}^{-1} A \Phi_{k-1}^{-1} A^{T} C^{T} \times\left[I+\lambda_{k-1}^{-1} C A \Phi_{k-1}^{-1} A^{T} C^{T}\right]^{-1}
$$

Calculation of the inverse of matrix $\Phi_{k}$

$$
\Phi_{k}^{-1}=\lambda_{k-1}^{-1} A \Phi_{k-1}^{-1} A^{T}-\lambda_{k-1}^{-1} K_{k} C A \Phi_{k-1}^{-1} A^{T}
$$

Calculation of the matrix $\mathrm{S}_{k}$

$$
\begin{aligned}
S_{k}= & \lambda_{k}^{-1}\left(I-K_{k} C\right) A S_{k-1} A^{T}\left(I-C^{T} K_{k}^{T}\right) \\
& -\lambda_{k}^{-1} \Phi_{k}^{-1}+\lambda_{k}^{-1} K_{k} K_{k}^{T}
\end{aligned}
$$

Calculation of the inverse of matrix $\psi_{k}$

$$
\psi_{k}=\left(A-K_{k} C A\right) \psi_{k-1}+S_{k} C^{T} \xi_{k}
$$

States (a-posteriori) estimates

$$
\hat{x}_{k}=A \hat{x}_{k-1}+K_{k} \xi_{k}
$$

Output estimate ( $y_{k}$ is the equalized signal)

$$
\hat{y}_{k}=C \hat{x}_{k}
$$

Calculation of the estimation error for performance analysis

$$
e_{k}=y_{k}-\hat{y}_{k}
$$

\section{State Space Model of Adaptive Equalizer}

We now describe the state space model used to implement the equalization algorithms discussed in the previous sections. The constant acceleration model has been used as state space 
model of the equalizer. The constant acceleration model as given in [5] and [6] as;

$$
\begin{gathered}
A=\left[\begin{array}{ccc}
1 & T & T^{2} / 2 \\
0 & 1 & T \\
0 & 0 & 1
\end{array}\right] \\
C=\left[\begin{array}{lll}
1 & 0 & 0
\end{array}\right]
\end{gathered}
$$

where $T$ is the time period of the actual system which is to be equalized. This model is actually for the unforced system, so there is a modification in matrix $\mathrm{C}$ for the case of the forced system as given in [6] and [7].

Furthermore, since the order of the channel $\left(12^{\text {th }}\right.$ order filter in our case) is unknown to the equalizer so the issue of model uncertainty is also present which is discussed in the next section. However if we consider the critically fitted case when the order of equalizer is equal to the order of the underlying unknown channel i.e. equal to 12 , then the matrices $\mathrm{A}$ and $\mathrm{C}$ are given as;

$$
\begin{aligned}
& A=\operatorname{diag}\left\{\left[\begin{array}{ccc}
1 & T & T^{2} / 2 \\
0 & 1 & T \\
0 & 0 & 1
\end{array}\right]\right\}, \quad i=1,2,3,4
\end{aligned}
$$

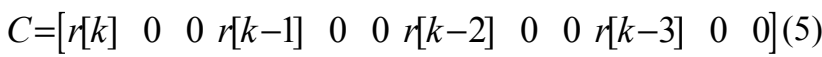

\section{Simulation Results}

In this section, we present the results of computer simulations performed in MATLAB. We consider the whole scenario under the two cases namely time invariant and time varying wireless channels given in Section II.

\section{A. Case I}

In this case, the simulations are performed for the tracking of a signal applied at the input of time invariant channel. Both the SSRLS and SSRLSWAM algorithms are initialized by the regularization term for $\delta=0.1$. The signal is observed in the discrete domain after sampling at $T=0.1 \mathrm{~s}$. For SSRLS, the forgetting factor is taken 0.95 and the same value is used for initialization of the time varying forgetting factor in SSRLSWAM. The learning rate parameter $(\alpha)$ is taken to be 0.0005 .

We use Ensemble Averaged Squared Estimation Error (EASEE) as a performance measure to compare different cases and sub-cases of SSRLS and SSRLSWAM. This performance measure i.e. EASEE is based on the ergodicity i.e. to approximate the ensemble average (true mean) of the estimation error; we take many realizations of the estimation error and then find the time average of the squared estimation error.

Fig. 1 depicts the simulation results for noise variance 0.1 whereas Fig. 2 shows the results for the time invariant case for noise variance of 0.01 . It can be seen from Fig. 1 and Fig. 2 that SSRLSWAM outperforms SSRLS in both the cases but the improvement is more in Fig. 2. So, it can be concluded that SSRLSWAM shows better performance as compared to SSRLS for time invariant channel especially for high SNRs.
Fig. 3 depicts the simulation results when the equalizer model order is made lesser than the channel model order. In our simulation, we consider an underfitted equalizer model of $6^{\text {th }}$ order for the constant acceleration model discussed in the previous section. It is clear that in the presence of such model uncertainties, SSRLSWAM again outperforms SSRLS even for low SNRs. This result affirms the effectiveness of SSRLSWAM for practical scenarios in which the model of underlying wireless channel is unknown to the equalizer.

\section{B. Case II}

In this case, the simulations are performed for the tracking of signal applied at the input of time varying channel i.e. the channel $\left(12^{\text {th }}\right.$ order FIR filter in our case) weights are not constant, rather they are continuously changing with time. We have implemented this time variance in the channel in the way such that the weights are changing in sinusoidal way at very slower rate as compared to the input at every time index i.e. at every recursion.
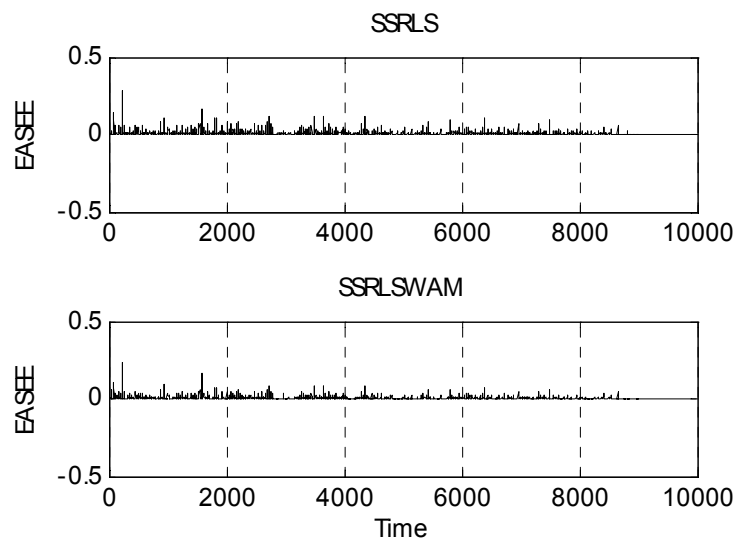

Fig. 1. Adaptive channel equalization for $\sigma_{\mathrm{v}}{ }^{2}=0.1$ (time invariant channel)
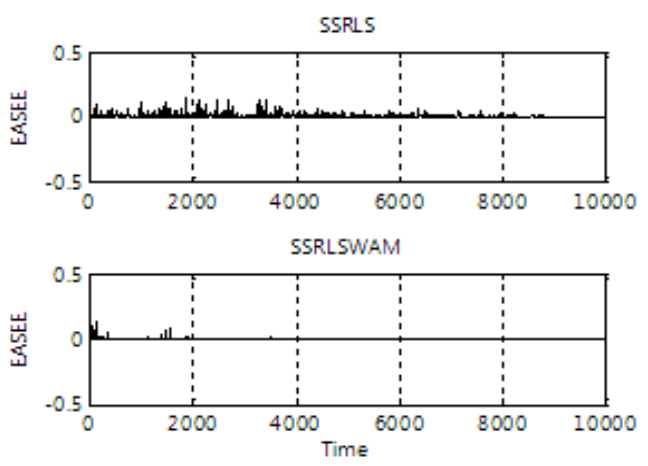

Fig. 2. Adaptive channel equalization for $\sigma_{\mathrm{v}}{ }^{2}=0.01$ (time invariant channel)
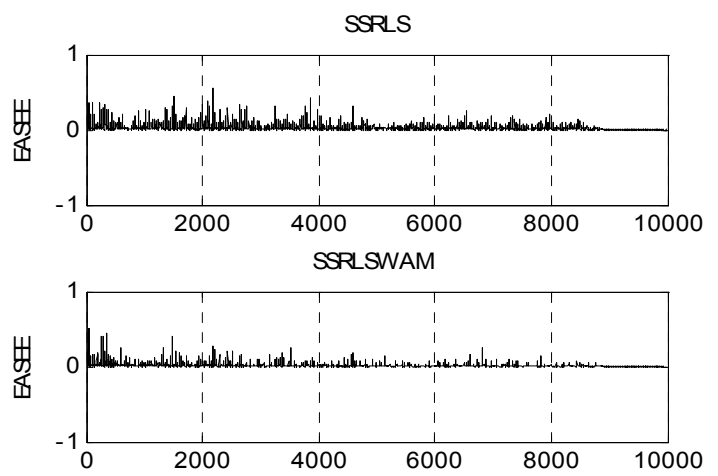

Fig. 3. Adaptive channel equalization for $\sigma_{\mathrm{v}}{ }^{2}=0.1$ (time invariant channel) and $6^{\text {th }}$ order equalizer (underfitted) 
The corresponding plots of EASEE for SSRLS and SSRLSWAM with the noise variances of 0.1 and 0.01 for time varying channel are shown in Fig. 4 and Fig. 5 respectively. The adaptation or tuning of the forgetting factor in case of noise variance of 0.1 is given in Fig. 6. By looking at the simulation results, it can be seen that in case of time invariant channel, the tracking performance of SSRLSWAM is a satisfactory as compared to SSRLS especially when noise variance is reduced. SSRLSWAM performs much better when we consider the case of model mismatch as depicted in Fig. 3, so we can say that SSRLSWAM is more valuable tool when model uncertainties are present. When considering time varying channel, SSRLSWAM is far better choice than standard SSRLS. The reason is the tuning of forgetting factor (Fig. 6) with the variations in the channel in SSRLSWAM.
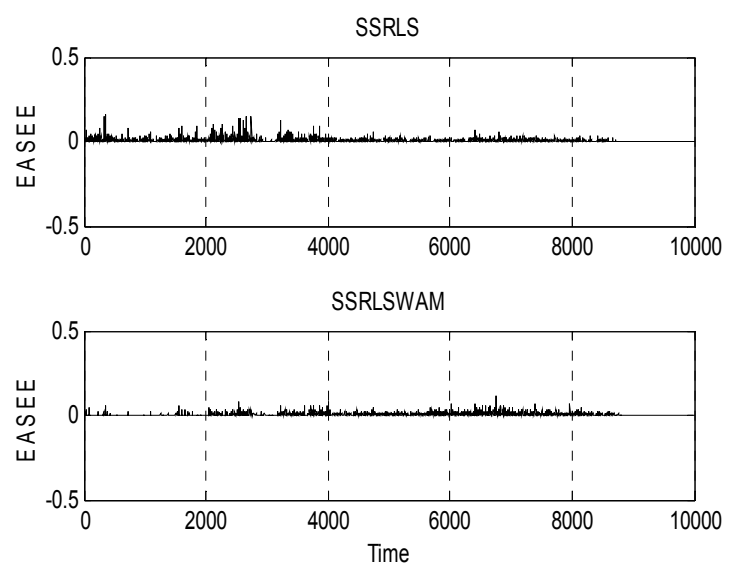

Fig. 4. Adaptive channel equalization for $\sigma_{\mathrm{v}}{ }^{2}=0.1$ (time varying channel)
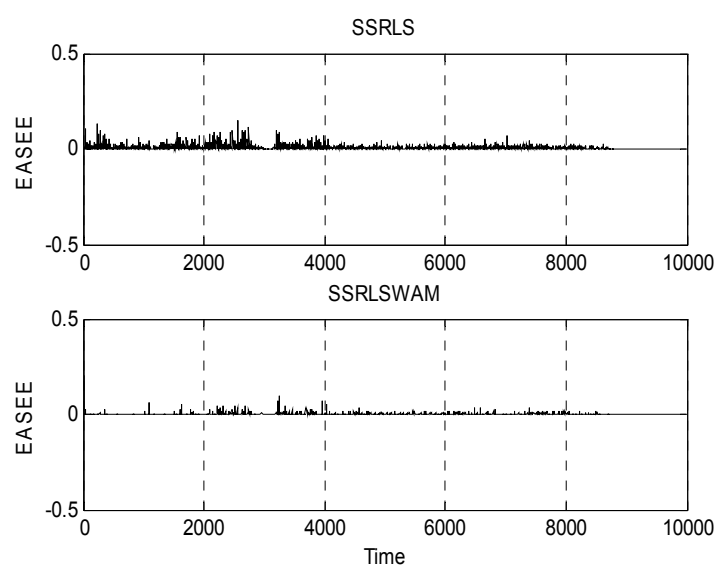

Fig. 5. Adaptive channel equalization for $\sigma_{\mathrm{v}}{ }^{2}=0.01$ (time varying channel)

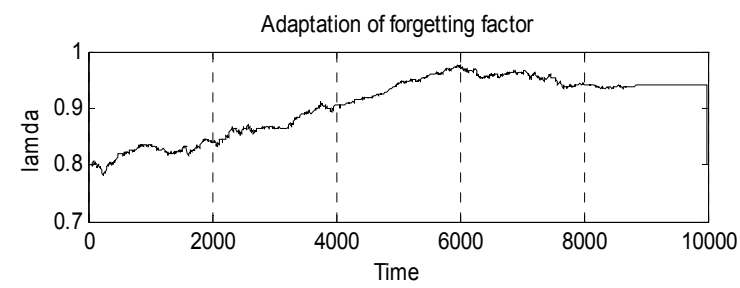

Fig. 6. Adaptation (tuning) of forgetting factor for $\sigma_{\mathrm{v}}{ }^{2}=0.1$ (time varying channel)

\section{CONCLUSION}

In this paper, we have performed adaptive channel equalization and compared the performance of SSRLS and SSRLSWAM. Both these algorithms are valuable tools in signal processing and adaptive filtering. Wireless channel with time varying coefficients has also been considered. It can be concluded that SSRLSWAM is more flexible filter due to the tuning of the forgetting factor in recursive manner. The Simulations results signify the capabilities of both SSRLS and SSRLSWAM. In the presence of model uncertainties and time varying environment, SSRLSWAM gives better tracking performance than SSRLS.

\section{REFERENCES}

[1] S. Haykin, Adaptive Filter Theory, $4^{\text {th }}$ edition, Prentice Hall, 2001, ch. 1, pp. 2-4.

[2] B. Sklar, Digital Communications, Fundamentals and Applications, $2^{\text {nd }}$ edition, Prentice Hall, 2002, ch. 3, pp. 152-153.

[3] M. B. Malik, "State-space recursive least-squares; Part I," Signal Process. Journal, vol. 84, pp. 1709-1718, 2004.

[4] M. B. Malik, "State-space recursive least-squares with adaptive memory," Signal Process. Journal, vol. 86, pp. 1365-1374, 2006.

[5] M. B. Malik, "State-space recursive least-squares," PhD Dissertation, Dept. of Elect. Engg., College of EME, National University of Sciences and Technology, Pakistan, 2004.

[6] M. B. Malik and M. Salman, "State-space least mean square," Digital Signal Processing, vol. 18, pp. 334-345, 2008.

[7] M. B. Malik, H. Qureshi, and R. A. Bhatti, "Tracking of linear time varying systems by state-space RLS," in Proc. of the IEEE International symposium on Circuits and System, 2004, vol. 3, pp. 305-308.

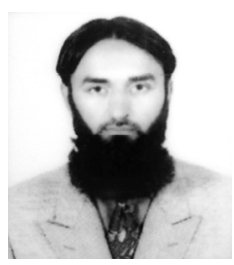

Muhammad Zeeshan completed his B.Sc. in Electrical Engineering from University of Engineering and Technology (UET), Taxila, Pakistan in 2008 and M.Sc. in Electrical Engineering from National University of Sciences and Technology (NUST), Pakistan in 2010. Since July 2010, he has been working as Senior Design Engineer in Center for Advanced Research in Engineering (CARE), Pakistan, where he has been involved in many research projects related to Software Defined Radio and Spread Spectrum technology. He is also a graduate student member of IEEE. Currently, he is working towards his Ph.D. in Electrical Engineering from National University of Sciences and Technology (NUST), Pakistan. His research interests include spread spectrum based wireless communications, synchronization techniques and digital design of communication systems.

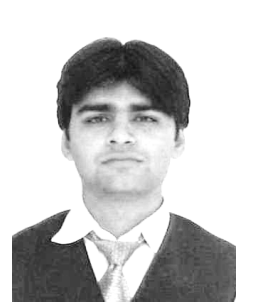

Ihsan Ullah completed his B.Sc. in Electrical Engineering from COMSATS Institute of Information Technology, Abbottabad in 2008 and M.Sc. in Electrical Engineering from National University of Sciences and Technology (NUST), Pakistan in 2010. From 2010 to June 2012, he was a faculty member in the Department of Electrical Engineering at COMSATS Institute of Information Technology, Abbottabad. Currently he is working towards his $\mathrm{Ph} . \mathrm{D}$. in Electrical Engineering from Hanyang University, South Korea. His research interests include wireless communications, and digital system design. 